\title{
Bifurcation Dynamics of a Particle-Encapsulating Droplet in Shear Flow
}

\author{
Lailai Zhu ${ }^{1,2,3,{ }^{*}}$ and François Gallaire ${ }^{1}$ \\ ${ }^{1}$ Laboratory of Fluid Mechanics and Instabilities, Ecole Polytechnique Fédérale de Lausanne, Lausanne CH-1015, Switzerland \\ ${ }^{2}$ Department of Mechanical and Aerospace Engineering, Princeton University, Princeton, New Jersey 08540, USA \\ ${ }^{3}$ Linné Flow Centre and Swedish e-Science Research Centre (SeRC), KTH Mechanics, Stockholm SE-10044, Sweden
}

(Received 31 March 2017; published 9 August 2017)

\begin{abstract}
To understand the behavior of composite fluid particles such as nucleated cells and double emulsions in flow, we study a finite-size particle encapsulated in a deforming droplet under shear flow as a model system. In addition to its concentric particle-droplet configuration, we numerically explore other eccentric and time-periodic equilibrium solutions, which emerge spontaneously via supercritical pitchfork and Hopf bifurcations. We present the loci of these solutions around the codimension-two point. We adopt a dynamic system approach to model and characterize the coupled behavior of the two bifurcations. By exploring the flow fields and hydrodynamic forces in detail, we identify the role of hydrodynamic particle-droplet interaction which gives rise to these bifurcations.
\end{abstract}

DOI: 10.1103/PhysRevLett.119.064502

Droplets, capsules, and vesicles in flow often exhibit interestingly rich dynamics even in the linear shear flow [1-9]. Despite substantial work on the dynamics of these soft systems enclosing homogeneous fluids, limited effort has been directed to studying their behavior when they include an internal structure. However, such a configuration is common in nature and engineering applications: cells like leukocytes and megakaryocytes contain a nucleus of up to $50 \%-80 \%$ of themselves in volume [10]; double emulsions playing an important role in chemical and pharmaceutical engineering are featured with a coreshell geometry [11-13]; droplet-based encapsulation for high-throughput biological assays utilizes droplets as microchambers to compartment cells for analysis at the single-cell level, where the cell size can be comparable to the droplet size in certain applications [14-16].

These fluid particles are characterized by complex hydrodynamic interactions between the internal structures and the external interface. Few works conducted for nucleated model cells in shear [17-20] all assumed their compound structures to be concentric, preserving the rotational symmetry of order $2(C 2)$ about the $y$ axis and reflection symmetry about the $y=0$ shear plane [see Fig. 1(a)]. The symmetries do hold for a single shear-driven deformable particle that attains a steady ellipsoidal shape undergoing tank-treading motion [21-23]. Yet, they are not guaranteed in the presence of an internal structure.

In this Letter we focus on the stability of the concentricity of composite fluid particles. By considering a droplet encaging a spherical particle as a model system, we formulate the following questions: will the composite structures remain concentric? How does the dynamics depend on interfacial tension and particle size? What is the role of the hydrodynamic interaction?

We begin our discussion by presenting 3D hydrodynamic simulations of a compound particle droplet subjected to unbounded shear $\mathbf{U}^{\infty}=\mathbf{G} \cdot \mathbf{x}$, in the creeping flow regime, where the only nonzero component $G_{x z}=\dot{\gamma}$ represents the shear rate [Fig. 1(a)]. The incompressible Stokes equations are solved by a boundary integral method [24]. The immiscible Newtonian fluids inside and outside the droplet have the same viscosity $\eta$; its surfactant-free interface has a uniform surface tension $\sigma$. The particle with a no-slip surface freely translates and rotates, experiencing zero hydrodynamic force and torque. The droplet interface satisfies the standard stress balance condition $[35,36]$. The radii of the particle and the undeformed droplet are $a$ and $R$, respectively; the size ratio is denoted by $\alpha=a / R$ with $\alpha \in(0,1)$. The capillary number $\mathrm{Ca}=\eta \dot{\gamma} R / \sigma$ indicates the ratio between viscous forces and capillary forces, limited to the regime without droplet breakup. All length scales are scaled by $R$.

We initially displace the particle away from the droplet center by a perturbative offset $\mathbf{d}=\left(d_{x}, d_{y}, d_{z}\right)$, then focusing on the time evolution of $d_{y}$ and $d_{x z}=\sqrt{d_{x}^{2}+d_{z}^{2}}$ representing the spanwise and in-plane displacements,

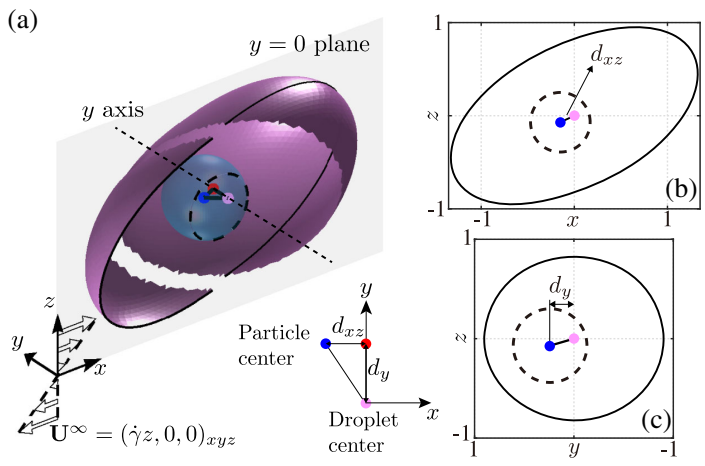

FIG. 1. (a) Sketch: a spherical particle moving inside a droplet under shear. A snapshot of the composite system cut by the $y=0$ (b) and $x=0$ (c) plane. 

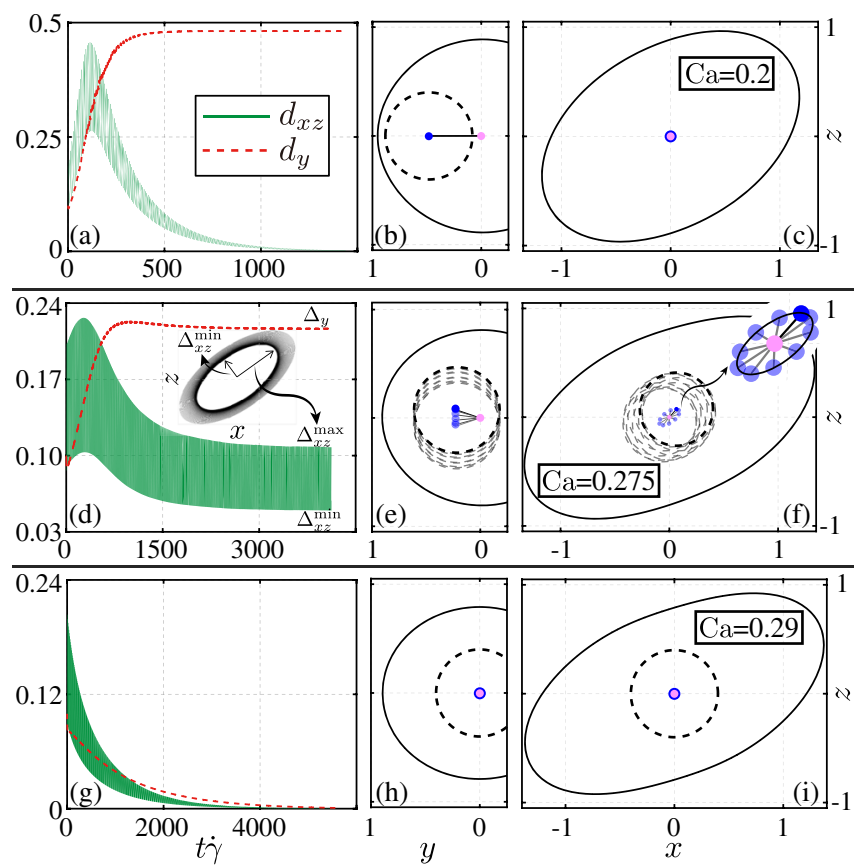

FIG. 2. Evolution of the in-plane and spanwise displacements $d_{x z}$ (green) and $d_{y}$ (red) between the centers of particle (blue) and droplet (pink), for size ratio $\alpha=0.4$ and $\mathrm{Ca}=0.2$ (first row), 0.275 (second row), and 0.29 (third row). The second (third) column shows the profile of the composite system at equilibrium, cut by $x=0(y=0)$ plane. The particle position is shown at different instants within a period for $\mathrm{Ca}=0.275$, the insets of (d) and (f) display the limit cycle solution.

respectively. $\Delta_{y}$ and $\Delta_{x z}$ denote their equilibrium values when the system reaches a steady or time-periodic state.

We show the evolution of displacements in Fig. 2, presenting three typical $\mathrm{Ca}$-dependent scenarios for a particle of size ratio $\alpha=0.4$ (see Supplemental Material videos [24]). When $\mathrm{Ca}=0.2$, the in-plane displacement $d_{x z}$ decays asymptotically to zero after a transient growth while the spanwise offset $d_{y}$ increases to a saturated value $\Delta_{y} \approx 0.48$ indicating the broken reflection symmetry. The particle rotates steadily near the lateral edge of the droplet interface [Fig. 2(b)]. Increasing Ca to $0.275, \Delta_{y}$ decreases to 0.21 approximately, while $d_{x z}$ reaches a time-periodic equilibrium cycle with a maximum of $\Delta_{x z}^{\max } \approx 0.11$ and a minimum of $\Delta_{x z}^{\min } \approx 0.04$. The particle follows an orbital trajectory on the $y=\Delta_{y}$ plane as it reaches a limit cycle solution in the $(x, z)$ space [Figs. 2(d), 2(f)], implying that the $C 2$ symmetry and time invariance are also broken. At $\mathrm{Ca}=0.29$, the system recovers steadiness and concentricity, $\Delta_{y}=\Delta_{x z}=0$. These scenarios suggest the appearance of bifurcating solutions by reducing $\mathrm{Ca}$ : above a critical value $\mathrm{Ca}_{c}(\alpha)$, the composite system stays concentric, corresponding to a stable fixed point solution; it bifurcates across $\mathrm{Ca}_{c}(\alpha)$ towards a steady spanwise migration (SM) and/or in-plane orbiting (IPO) motion.

An investigation spanning the $(\mathrm{Ca}, \alpha)$ space further reveals that these two modes, i.e., SM and IPO, appear spontaneously through supercritical pitchfork and Hopf bifurcations, respectively. To study the evolution of the two modes individually, we perform decoupled simulations with kinematic constraints of either $d_{x z}=0$ (pure SM) or $d_{y}=0$ (pure IPO). Their corresponding equilibrium displacements $\tilde{\Delta}_{y}$ and $\tilde{\Delta}_{x z}^{\min / \max }$ are shown in Fig. 3. For all $\alpha$, we observe $\tilde{\Delta}_{y}$ decreases with $\mathrm{Ca}$, becoming zero when Ca exceeds a critical value $\mathrm{Ca}_{c}(\alpha)$, so does $\tilde{\Delta}_{x z}^{\min / \max }$. They both vary quadratically in the vicinity of their corresponding $\mathrm{Ca}_{c}(\alpha)$. This is confirmed by the linear fitting of $\tilde{\Delta}_{y}$ and, $\tilde{\mathcal{A}}_{x z}=\tilde{\Delta}_{x z}^{\max }-\tilde{\Delta}_{x z}^{\min }$ indicating the oscillating amplitude, versus $\left[\mathrm{Ca}_{c}(\alpha)-\mathrm{Ca}\right]^{1 / 2}\left[\right.$ Fig. 3(d)], where $\mathrm{Ca}_{c}(\alpha)$ is obtained simultaneously. The successful fitting passing through the origin verifies the emergence of the two bifurcations. It is worth pointing out that broken reflection symmetry by SM is indeed the signature of pitchfork bifurcation, so as broken time invariance by IPO is of Hopf bifurcation.

Let us return to the constraint-free cases, where the nonlinear interaction of the two modes results in a more complex dependence of the equilibrium solutions on $\mathrm{Ca}$ and $\alpha$ (Fig. 3). For $\alpha=0.3$, the in-plane amplitudes (cyan) reach their maxima around $\mathrm{Ca}=0.3$, from where they decrease almost linearly (quadratically) with decreasing (increasing) $\mathrm{Ca}$. In their quadratic parts, the coupled (cyan) and decoupled (green) amplitudes overlap in the vicinity of their common critical point. In contrast, the spanwise
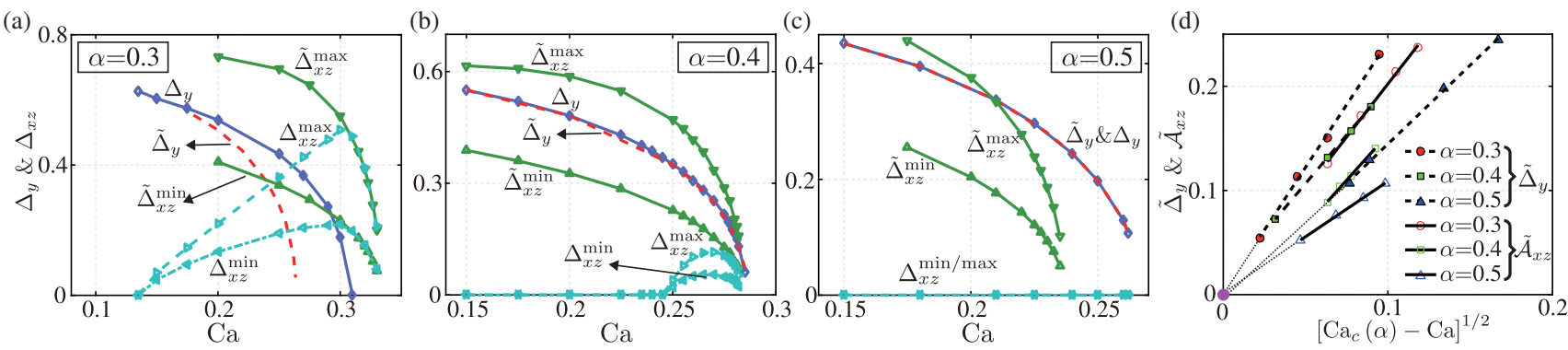

FIG. 3. Equilibrium displacements $\Delta_{y}, \Delta_{x z}^{\min / \max }$ versus Ca for $\alpha=0.3$ (a), 0.4 (b), and 0.5 (c); $\sim$ denotes their counterparts of the decoupled simulations. (d) Linear fitting of $\tilde{\Delta}_{y}$ and $\tilde{\mathcal{A}}_{x z}=\tilde{\Delta}_{x z}^{\max }-\tilde{\Delta}_{x z}^{\min }$ versus $\left[\mathrm{Ca}_{c}(\alpha)-\mathrm{Ca}\right]^{1 / 2}$. 
(a)

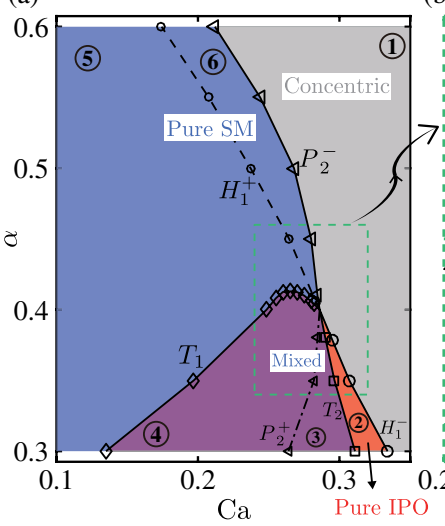

(b)

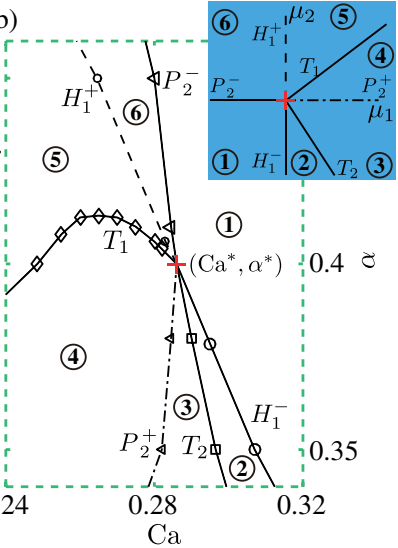

FIG. 4. (a) Parametric portrait displaying the loci of four solution types in the $(\mathrm{Ca}, \alpha)$ space. (b) Close-up of (a) in the vicinity of the codimension-two point $\left(\mathrm{Ca}_{*}, \alpha_{*}\right)$ from where six bifurcation curves originate. The inset shows the bifurcation topology in the $\left(\mu_{1}, \mu_{2}\right)$ space.

offsets (purple) are larger than those of the decoupled cases (red). For $\alpha=0.4$, the spanwise offsets coincide precisely with the decoupled counterparts for all $\mathrm{Ca}$, while the inplane amplitudes exhibit nonmonotonic $\mathrm{Ca}$ dependence as for $\alpha=0.3$ and they are below the decoupled values. For $\alpha=0.5$, perfect coincidence between the spanwise offsets also holds as in the $\alpha=0.4$ case, while $\Delta_{x z}^{\min / \max } \equiv 0$ for all $\mathrm{Ca}$; i.e., the Hopf bifurcation is inhibited.

The complexity is better unraveled by the parametric portrait quartering the $(\mathrm{Ca}, \alpha)$ parameter space into the following solution types (Fig. 4): "concentric" implying the absence of both modes, "pure IPO," "pure SM," and "mixed" indicating the coexistence of both modes. A codimension-two point $\left(\mathrm{Ca}_{*}, \alpha_{*}\right) \approx(0.286,0.4)$ is pinpointed at the intersection of the two marginal curves $H_{1}^{ \pm}$ (circle) and $P_{2}^{ \pm}$(triangle) which correspond to the Hopf and pitchfork bifurcations, respectively. Other branches bifurcating from this point are $T_{1}$ separating pure SM and mixed, $T_{2}$ separating pure IPO and mixed. Note ${ }^{+}$and ${ }^{-}$denote the upper and lower branches of the marginal curves.

We now interpret the bifurcation in the neighborhood of the codimension-two point $\left(\mathrm{Ca}_{*}, \alpha_{*}\right)$ based on a normalform analysis. By coupling the amplitude equations of the Hopf and pitchfork bifurcations, we obtain a normal form similar to that of the Hopf-Hopf bifurcation in Ref. [37] where the amplitudes are independent of phase evolution. Denoting the square of the in-plane and spanwise amplitudes by $\rho_{1}=\left(\Delta_{x z}^{\max }-\Delta_{x z}^{\min }\right)^{2}$ and $\rho_{2}=\Delta_{y}^{2}$, the truncated amplitude system is expressed as

$$
\begin{aligned}
& \dot{\rho}_{1}=\rho_{1}\left(\mu_{1}+p_{11} \rho_{1}+p_{12} \rho_{2}\right), \\
& \dot{\rho}_{2}=\rho_{2}\left(\mu_{2}+p_{21} \rho_{1}+p_{22} \rho_{2}\right),
\end{aligned}
$$

where $\mu_{i}$ represent the linear growth rates of the individual modes and $p_{i j}$ the nonlinear coupling coefficients. Because of the supercritical nature of the two bifurcations, $p_{11}<0$ and $p_{22}<0$. Physically, the amplitudes tend to asymptotic values with decreasing $\mathrm{Ca}$ (see Fig. 3) owing to the confinement of droplet. Because $p_{11} p_{22}>0$, our problem is in the category of the so-called simple cases, for which we have neglected $\rho_{1} \rho_{2}^{2}$ and $\rho_{2} \rho_{1}^{2}$ without changing the bifurcation topology [37]. By introducing new phase variables $\xi_{1}=-p_{11} \rho_{1}$ and $\xi_{2}=-p_{22} \rho_{2}$, we obtain

$$
\begin{aligned}
& \dot{\xi}_{1}=\xi_{1}\left(\mu_{1}-\xi_{1}-\theta \xi_{2}\right), \\
& \dot{\xi}_{2}=\xi_{2}\left(\mu_{2}-\delta \xi_{1}-\xi_{2}\right),
\end{aligned}
$$

where $\theta=p_{12} / p_{22}$ and $\delta=p_{21} / p_{11}$. Applying at leading order the affine transformation

$$
\begin{aligned}
& \mu_{1}=k_{1}\left(\mathrm{Ca}-\mathrm{Ca}_{*}\right)-\left(\alpha-\alpha_{*}\right), \\
& \mu_{2}=C\left[k_{2}\left(\mathrm{Ca}-\mathrm{Ca}_{*}\right)-\left(\alpha-\alpha_{*}\right)\right],
\end{aligned}
$$

in the vicinity of $\left(\mathrm{Ca}_{*}, \alpha_{*}\right)$, we map the parameter space from $(\mathrm{Ca}, \alpha)$ to $\left(\mu_{1}, \mu_{2}\right)$ [inset of Fig. 4(b)], where $k_{1} \approx$ -2.3 and $k_{2} \approx-40$ denote the slope of $H_{1}$ and $P_{2}$ curves at $\left(\mathrm{Ca}_{*}, \alpha_{*}\right)$, with $C \approx 0.043$ derived from the growth rate of $\rho_{i}$. The slopes of $T_{1}$ and $T_{2}$ further determine $\theta \approx 0.71$ and $\delta \approx-0.69$. The parametric portrait, therefore, corresponds to case III described in Ref. [37], characterized by six regions: (1) corresponding to concentric; (2) to pure IPO; (3) and (4) to mixed separated by $P_{2}^{+}$; (5) and (6) to pure SM separated by $H_{1}^{+}$[24].

The parametric portrait and normal form both reveal nonlinear mode interactions as a fingerprint of the present bifurcation. In the absence of SM, IPO appears stably in regions (2)-(5) but it is suppressed due to the nonlinear interaction with SM, as reflected by the phase portraits of (3)-(5) all including an unstable saddle-node equilibrium $\left(\mu_{1}, 0\right)$, as well as by the sign of $\theta$. Consequently, pure IPO only survives in (2). Besides, without IPO, pure SM is stable in regions (4-@6, while IPO promotes SM to expand its locus further to (3) that indeed involves a stable equilibrium $\left(\mu_{1}, \mu_{2}\right)$. This promotion results from the sign of $\delta$.

We next reveal the mechanisms underlying the bifurcations, first focusing on $H_{1}$ and $P_{2}$ separately. The shear flow can be decomposed into a rotational and extensional part, and we found that the former alone does not contribute to the particle's cross-stream motions. The eccentricity is mostly driven by the extensional part $\mathbf{U}_{E}^{\infty}(\mathbf{x})=\mathbf{E} \cdot \mathbf{x}$ with $E_{x z}=E_{z x}=\dot{\gamma} / 2$. For a system with imposed concentricity in $\mathbf{U}_{E}^{\infty}(\mathbf{x})$, Fig. 5 displays the droplet-induced disturbance flows $\hat{\mathbf{u}}_{d}$ on $y=0$ (a) and $x=z$ (b) plane, which preserve reflection symmetries about each other. The major and minor axis of the ellipsoid-shaped droplet lies on the $x=$ $\pm z$ plane. The disturbance flow is induced to satisfy zero normal velocities on the interface. On the shear plane, it approaches (leaves) the origin along the major (minor) axis. It resembles the stagnation point flow, where the origin is 

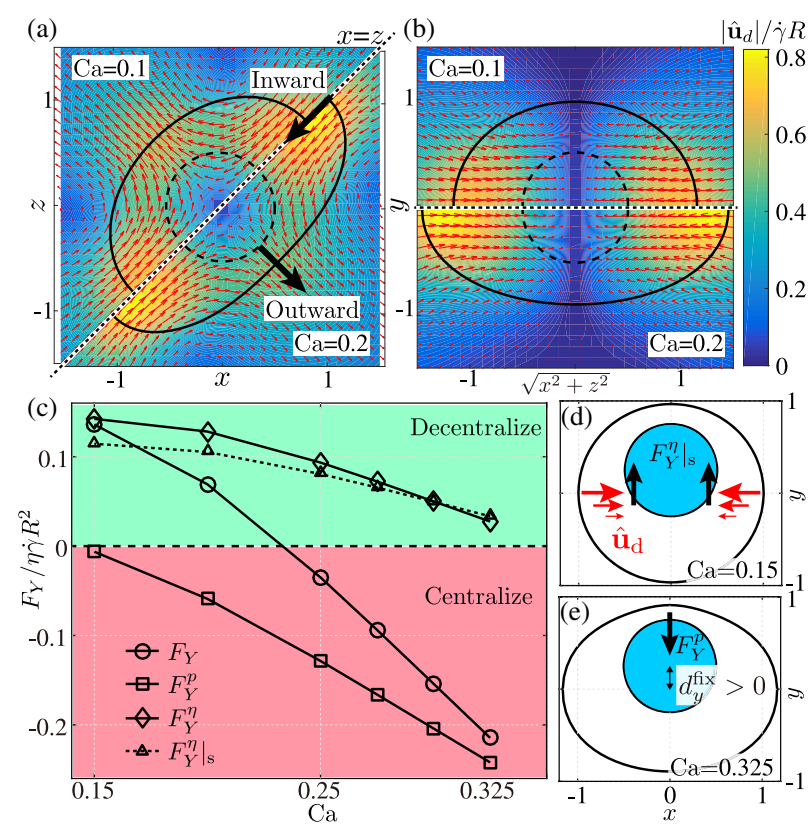

FIG. 5. Droplet-induced disturbance flow $\hat{\mathbf{u}}_{d}$ of concentric systems in the extensional flow, shown on the $y=0$ (a) and $x=z$ (b) plane, for $\alpha=0.5, \mathrm{Ca}=0.1$ and 0.2. (c) Hydrodynamic force $F_{Y}(\mathrm{Ca})$ exerted on the particle with a fixed offset $\left(0, d_{y}^{\text {fix }}>0,0\right)$ inside the droplet under shear; $F_{Y}^{p}$ and $F_{Y}^{\eta}$ denote the pressure and viscous parts of $F_{Y}$, respectively, and $\left.F_{Y}^{\eta}\right|_{S}$ the shear force of $F_{Y}^{\eta}$. The composite profile is shown on the $z=0$ plane for $\mathrm{Ca}=0.15$ (d) and 0.325 (e).

kinematically unstable. This initiates the in-plane motion and indeed the particle moves along the minor axis and eventually touches the droplet for any $\mathrm{Ca}$ if we free its inplane motion. This scenario is altered by the rotational flow, which relocates the particle between the two axes cyclically. Consequently, it is centralized (decentralized) by the inward (outward) flow after every relocation. The inward and outward flows roughly balance at $\mathrm{Ca}=0.1$, while the former dominates the latter at $\mathrm{Ca}=0.2$, hence overcoming the kinematic instability and leading to a concentric preference. This might explain the quenching of IPO when $\mathrm{Ca}$ increases across the marginal $H_{1}$ curve. On the $x=z$ plane [Fig. 5(b)], the flow resembles a parallel compressional flow that reaches the maximum strength at $y=0$ and weakens in the $\pm y$ directions. When the particle undergoes a spanwise $d_{y}$ perturbation (say $d_{y}>0$ ), it experiences the strongest compression on its lower part and the $y$ gradient of that compressional flow will produce a viscous shear force in the spanwise direction (see discussion below) which further amplifies this perturbation, triggering the pitchfork bifurcation. Note that the flow on the $x=-z$ plane may conversely help centralize the particle, yet, it is weaker for any $\mathrm{Ca}>0$. Moreover, we conduct simulations fixing a certain spanwise offset $d_{y}^{\text {fix }}>0$ with $d_{x}=d_{z}=0$ for the shear flow, recording the spanwise hydrodynamic forces $F_{Y}=F_{Y}^{p}+F_{Y}^{\eta}$ on the particle [Fig. 5(c)], where $F_{Y}^{p}$
$\left(F_{Y}^{\eta}\right)$ represents the pressure (viscous) contribution which centralizes (decentralizes) the particle. As shown, the viscous shear force $\left.F_{Y}^{\eta}\right|_{s}$ accounts for the major contribution to $F_{Y}^{\eta}$, supporting the above arguments of compression-induced viscous destabilization. The pressure force becomes stronger with $\mathrm{Ca}$ and dominates the viscous part when $\mathrm{Ca}$ exceeds a critical value. In fact, the droplet with larger Ca displays a lateral protrusion accompanying a local curvature increase [Fig. 5(e)], generating a stronger pressure to center the particle. This clarifies why SM vanishes when $\mathrm{Ca}$ crosses the $P_{2}$ curve. Upon having elucidated $H_{1}$ and $P_{2}$ bifurcations individually, we comment on $T_{1}$ and $T_{2}$ which involve mode interactions. The trajectory of IPO lies on $y=\Delta_{y}$ plane, hence bounded within a circular orbit of radius $\left(1-\Delta_{y}^{2}\right)^{1 / 2}-\alpha$ approximately, because the particle simply cannot penetrate the droplet. The SM mode hence suppresses the IPO mode due to the confinement; a larger $\Delta_{y}$ and/or $\alpha$ naturally shrinks the orbital displacement $\Delta_{x z}$ to be zero, when entering (5) across $T_{1}$. On the contrary, the emergence of $T_{2}$ reflects the promotive effect of IPO on SM. Regarding this, we may surmise that when the particle starts orbiting on the $y=0$ plane, it comes closer to the droplet interface; therefore, it suffers a greater compressional flow [as indicated by Fig. 5(b)] which results in stronger destabilizing viscous shear forces.

In summary, we have presented in this Letter hydrodynamic-interaction-meditated dynamics of a particle inside a droplet in steady shear flow. We have numerically discovered several equilibrium solutions where the composite system exhibits spontaneous symmetry breaking and unsteady dynamics rising through supercritical pitchfork and Hopf bifurcations; the particle can execute spanwise migratory and/or in-plane orbital movement. The bifurcations are partially attributed to the dropletinduced disturbance flow characterized by a kinematically unstable stagnation point. We have performed a normalform analysis to delineate the interplay between bifurcations, revealing the suppression of the Hopf bifurcation by migration and promotion of the pitchfork bifurcation by orbital motion. The interplay can be rationalized by the geometric confinement and the disturbance flow.

It is worth pointing out that the bifurcation dynamics might not be directly generalized to the two commonly adopted models of cells, capsule, and vesicle featured with elastic membranes. The in-plane elastic stresses developed on the interface might considerably suppress the interior flow that influences the inclusion dynamics.

We envision that our results might potentially inspire new approaches of "hydrodynamic centering" composite systems like emulsions to obtain a uniform shell in addition to electric centering methods $[38,39]$, or vice versa, using the hydrodynamic effect to generate emulsions with predesigned nonuniform shell thickness [40] for programmed release of substances. We hope our study will motivate experiments in these directions. We plan to address in our 
future work the influences of nonuniform shear, geometric features, and confinement of the setup, which are all relevant for practical applications.

The authors thank Jan Guzowski, Jérôme Hoepffner, Philippe Meliga, Arne Nordmark, and Howard A. Stone for useful discussions. The computer time is provided by the Swiss National Supercomputing Centre (CSCS) under project ID s603 and by SNIC (Swedish National Infrastructure for Computing). A VR International Postdoc Grant from Swedish Research Council "201506334" (L. Z.) and an ERC starting grant "SimCoMiCs 280117" (F. G.) are gratefully acknowledged.

*lailaizhu00@gmail.com

[1] H. A. Stone, Dynamics of drop deformation and breakup in viscous fluids, Annu. Rev. Fluid Mech. 26, 65 (1994).

[2] K. A. Smith, J. M. Ottino, and M. O. de la Cruz, Encapsulated Drop Breakup in Shear Flow, Phys. Rev. Lett. 93, 204501 (2004).

[3] V. Sibillo, G. Pasquariello, M. Simeone, V. Cristini, and S. Guido, Drop Deformation in Microconfined Shear Flow, Phys. Rev. Lett. 97, 054502 (2006).

[4] J. M. Skotheim and T. W. Secomb, Red Blood Cells and Other Nonspherical Capsules in Shear Flow: Oscillatory Dynamics and the Tank-Treading-to-Tumbling Transition, Phys. Rev. Lett. 98, 078301 (2007).

[5] T. Omori, Y. Imai, T. Yamaguchi, and T. Ishikawa, Reorientation of a Nonspherical Capsule in Creeping Shear Flow, Phys. Rev. Lett. 108, 138102 (2012).

[6] M. Kraus, W. Wintz, U. Seifert, and R. Lipowsky, Fluid Vesicles in Shear Flow, Phys. Rev. Lett. 77, 3685 (1996).

[7] C. Misbah, Vacillating Breathing and Tumbling of Vesicles under Shear Flow, Phys. Rev. Lett. 96, 028104 (2006).

[8] H. Noguchi and G. Gompper, Swinging and Tumbling of Fluid Vesicles in Shear Flow, Phys. Rev. Lett. 98, 128103 (2007).

[9] J. Deschamps, V. Kantsler, and V. Steinberg, Phase Diagram of Single Vesicle Dynamical States in Shear Flow, Phys. Rev. Lett. 102, 118105 (2009).

[10] M. L. Turgeon, Clinical Hematology: Theory and Procedures (Lippincott Williams \& Wilkins, Philadelphia, Pennsylvania, USA, 2005).

[11] H. A. Stone and L. Leal, Breakup of concentric double emulsion droplets in linear flows, J. Fluid Mech. 211, 123 (1990).

[12] A. Utada, E. Lorenceau, D. Link, P. Kaplan, H. Stone, and D. Weitz, Monodisperse double emulsions generated from a microcapillary device, Science 308, 537 (2005).

[13] J. Guzowski, S. Jakiela, P. M. Korczyk, and P. Garstecki, Custom tailoring multiple droplets one-by-one, Lab Chip 13, 4308 (2013).

[14] M. He, J. S. Edgar, G. D. Jeffries, R. M. Lorenz, J. P. Shelby, and D. T. Chiu, Selective encapsulation of single cells and subcellular organelles into picoliter- and femtoliter-volume droplets, Anal. Chem. 77, 1539 (2005).
[15] M. Chabert and J.-L. Viovy, Microfluidic high-throughput encapsulation and hydrodynamic self-sorting of single cells, Proc. Natl. Acad. Sci. U.S.A. 105, 3191 (2008).

[16] L. Mazutis, J. Gilbert, W. L. Ung, D. A. Weitz, A. D. Griffiths, and J. A. Heyman, Single-cell analysis and sorting using droplet-based microfluidics, Nat. Protoc. 8, 870 (2013).

[17] S. K. Veerapaneni, Y.-N. Young, P. M. Vlahovska, and J. Bławzdziewicz, Dynamics of a Compound Vesicle in Shear Flow, Phys. Rev. Lett. 106, 158103 (2011).

[18] B. Kaoui, T. Krüger, and J. Harting, Complex dynamics of a bilamellar vesicle as a simple model for leukocytes, Soft Matter 9, 8057 (2013).

[19] M. Levant and V. Steinberg, Complex Dynamics of Compound Vesicles in Linear Flow, Phys. Rev. Lett. 112, 138106 (2014).

[20] Z. Y. Luo, L. He, and B. F. Bai, Deformation of spherical compound capsules in simple shear flow, J. Fluid Mech. 775, 77 (2015).

[21] J. Rallison, The deformation of small viscous drops and bubbles in shear flows, Annu. Rev. Fluid Mech. 16, 45 (1984).

[22] U. Seifert, Configurations of fluid membranes and vesicles, Adv. Phys. 46, 13 (1997).

[23] D. Barthès-Biesel, Motion and deformation of elastic capsules and vesicles in flow, Annu. Rev. Fluid Mech. 48, 25 (2016).

[24] See Supplemental Material at http://link.aps.org/ supplemental/10.1103/PhysRevLett.119.064502 for the numerical methods and the validation, generic phase portraits of the parametric portrait, animations of the three typical scenarios shown in Fig. 2, which includes Refs. [2534].

[25] M. J. Lighthill, On the squirming motion of nearly spherical deformable bodies through liquids at very small reynolds numbers, Commun. Pure Appl. Math. 5, 109 (1952).

[26] J. R. Blake, A spherical envelope approach to ciliary propulsion, J. Fluid Mech. 46, 199 (1971).

[27] H. Lambs, Hydrodynamics, 6th ed. (Cambridge University Press, Cambridge, England, 1932).

[28] J. Happel and H. Brenner, Low Reynolds Number Hydrodynamics (Noordhoff Intl. Pub., Leyden, 1973).

[29] L. Zhu, E. Lauga, and L. Brandt, Low-Reynolds-number swimming in a capillary tube, J. Fluid Mech. 726, 285 (2013).

[30] A. Zinchenko and R. Davis, Emulsion flow through a packed bed with multiple drop breakup, J. Fluid Mech. 725, 611 (2013).

[31] G. Gallino, L. Zhu, and F. Gallaire, The stability of a rising droplet: an inertialess nonmodal growth mechanism, J. Fluid Mech. 786, R2 (2015).

[32] L. Zhu and F. Gallaire, A pancake droplet translating in a Hele-Shaw cell: lubrication film and flow field, J. Fluid Mech. 798, 955 (2016).

[33] S. Balay, W. D. Gropp, L. C. McInnes, and B. F. Smith, in Modern Software Tools in Scientific Computing, edited by E. Arge, A. M. Bruaset, and H. P. Langtangen (Birkhäuser Press, Cambridge, MA, USA, 1997), p. 163.

[34] S. Y. Reigh, L. Zhu, F. Gallaire, and E. Lauga, Swimming with a cage: low-Reynolds-number locomotion inside a droplet, Soft Matter 13, 3161 (2017). 
[35] $\boldsymbol{\Pi}_{1} \cdot \mathbf{n}-\boldsymbol{\Pi}_{2} \cdot \mathbf{n}=\sigma \mathbf{n}\left(\nabla_{S} \cdot \mathbf{n}\right), \quad$ where $\quad \boldsymbol{\Pi}_{1} \quad$ and $\quad \boldsymbol{\Pi}_{2}$ denote the total stress tensor corresponding to the carrier and droplet phase respectively, $\mathbf{n}$ the unit normal vector on the interface towards the carrier phase, and $\nabla_{S}=(\mathbf{I}-\mathbf{n n}) \cdot \nabla$ represents the surface gradient.

[36] L. G. Leal, Advanced Transport Phenomena: Fluid Mechanics and Convective Transport Processes (Cambridge University Press, Cambridge, England, 2007).

[37] Y. A. Kuznetsov, Elements of Applied Bifurcation Theory (Springer Science \& Business Media, New York, 2013), Vol. 112, p. 356.
[38] Z.-M. Bei, T. Jones, A. Tucker-Schwartz, and D. Harding, Electric field mediated droplet centering, Appl. Phys. Lett. 93, 184101 (2008).

[39] A. K. Tucker-Schwartz, Z. Bei, R. L. Garrell, and T. B. Jones, Polymerization of electric field-centered double emulsion droplets to create polyacrylate shells, Langmuir 26, 18606 (2010).

[40] Y. Hennequin, N. Pannacci, C. P. de Torres, G. TetradisMeris, S. Chapuliot, E. Bouchaud, and P. Tabeling, Synthesizing Microcapsules with Controlled Geometrical and Mechanical Properties with Microfluidic Double Emulsion Technology, Langmuir 25, 7857 (2009). 